\title{
Health@FirstHand (Fully featured, automated Epidemic Disease Management System)
}

\author{
K.M.P. D.R. Bandara \\ D.K.B. Dodanwala \\ H.M. Gunarathne \\ I.R.R.C.D. Ranabahu \\ Department of Computer Engineering, Faculty of Engineering, University of Peradeniya, Sri Lanka. \\ Contact e-mail address: moulyg@gmail.com
}

eHealth Sri Lanka 2010,1(suppl.1):S5

DOI: http://dx.doi.org/10.4038/sljbmi.v1i0.3537

Only the Abstract is available

\begin{abstract}
Introduction: In a situation where epidemic disease management such as Dengue is heavily discussed, it is important to do an efficient epidemic disease management, saving the valuable time to take action to prevent and reduce the spreading of the diseases.

General Objective: To develop a web based, automated and efficient software solution to the epidemic disease management system by introducing totally new components and enhancing the existing components in the system. Specific Objectives: 1. Helping doctors to identify the specific epidemic disease. 2. Giving past treatments for specific epidemic diseases expecting their success to decide the best treatment. 3. Analysing an area for epidemic diseases using maps and charts. 4. A prediction for an epidemic disease up to next two months. 5. Automated SMS and e-mail alerting. 6. Giving automatically generated reports monthly and annually.

Methodology: Developing the software using Microsoft Visual Studio 2008 (with Visual C\#) and Microsoft SQL Server as the DBMS. Bing maps and Microsoft Silverlight have been used to do the analysis using maps and charts, respectively. Results: Developed the expected software with the capability of having huge amounts of real time data. Confidentiality: Different levels of health officers have different access levels. The system ensures the speed and reliability. Conclusion: Health@FirstHand can provide a far better functionality and support in epidemic disease management rather than conventional, manual system. It saves time with real time data, quick analysis and alerting which is helpful in resource allocation, public awareness, etc.
\end{abstract}

Keywords - Dengue, epidemic disease, epidemic disease management system 